\title{
Novel treatments in Epilepsy guided by Genetic Diagnosis
}

\author{
Carla Marini ${ }^{1}$ and Maria Giardino ${ }^{1}$ \\ ${ }^{1}$ Ospedale Pediatrico G Salesi
}

May 12, 2021

\begin{abstract}
Identifying the optimal treatment based on specific aetiology of each patient is the main promise of precision medicine. In order to realize this promise researches and physicians must first identify the underlying cause; over the last 10 years, advances in genetics have made this possible for several monogenic epilepsies. At present through next generation techniques we can reach the precise genetic aetiology in 30 to $50 \%$ of genetic epilepsies beginning in the paediatric age. While committed in such gene hunting, progresses in the study of experimental models of epilepsy have also provided a better understanding of the mechanisms underlying the condition. Such impressive advances is already being translated into improving care, management and treatment of some patients. Identification of a precise genetic etiology can already direct physicians to prescribe treatments correcting specific metabolic defects avoid antiseizure medicines that can aggravate the pathogenic defect or select the drug that counteract the functional disturbance caused by the gene mutation. Personalized, tailored treatments should not just focus on how to stop seizures but possibly preventing their onset and cure the disorder often consisting of epilepsy and its comorbidities including cognitive, motor and behavior deficiencies. This review discusses the therapeutic implications following a specific genetic diagnosis and the correlation between genetic findings, pathophysiological mechanism and tailored seizure treatment emphasizing the impact on current clinical practice.
\end{abstract}

\section{Introduction}

Seizures are often the presenting and distressing symptom of epilepsies and syndromes including the fearsome developmental epileptic encephalopathies (DEE) (Scheffer et al 2017). Seizures are frequently the clinical sign that brings children to medical attention thus, the whole community of parents, professionals and scientists has always focused on how to stop seizures. Accordingly, studies on outcomes have also paid much attention on the treatment of seizures. Evidences from clinical practice and from animal models certainly vouch for a better developmental outcome when seizures are short, infrequent and possibly completely under control (Ragona et al 2011; Caraballo et al 2014; Meldrum, Horton 1973; Trinka et al 2015). Yet, epilepsy represents only a clinical manifestation that is often observed as part of complex neurodevelopmental disorders, including intellectual disability and autism. Thus, seizures are not the end of the story and comorbidities including motor, cognitive and behavioural impairments are equally relevant persisting beside and beyond seizures. In the ideal situation, a treating physician should aim to cure the whole diseases rather than some of the symptoms. Side effects produced by available antiseizure medications (ASM) add to the complexity of epilepsies and syndromes. In this complex scenario, the underlying aetiology certainly plays a leading role. The application of new methodologies including next generation sequencing (NGS), whole exome and genome sequencing (WES/WGS) has deeply transformed our understanding of the basis and pathophysiology of some epilepsies (Mei et al 2017, Dunn et al. 2018; Nabbout, Kuchenbuch 2020). We now know that the majority of epilepsies, especially those with infantile onset, have a genetic origin and hundreds of monogenic forms have been identified and dissected (Parrini et al 2017; Hebbar, Mefford 2020; Stödberg et al 2020; Symonds, McTague 2020). Ion channel gene including sodium, potassium and calcium represent the gene family most frequently associated to epilepsy (Parrini et al 2017; Stödberg et al 2020). Other genes have gradually emerged and have been associated with complex developmental disorders 
featuring seizures and revealing the pathogenic role of mutations affecting diverse pathways contributing to membrane excitability, synaptic plasticity, presynaptic neurotransmitter release, postsynaptic receptors, transporters, cell metabolism. Additional genes have relevant roles in many formative steps in early brain development, such as the proliferation and migration of neuronal precursors, dendritogenesis, synaptogenesis, cell and glial biology (Guerrini R, Noebels 2014; Szepetowski P. 2018)

Such impressive advances in knowledge has paved the way for a shift in the therapeutic management of patients from a population approach, based on epilepsy types and syndromes to, an individualized approach, where treatments could be targeted to genetically defined subgroups of individuals. The ideal individualized approach, in addition to the epilepsy type or syndrome, should take into consideration a combination of characteristics specific to the individual patient including age, race, sex and physiological parameters. This shift towards precision, or personalized, medicine will improve outcome and enable physicians to treat patients in a more targeted manner.

In the last few years, the scientific community, possibly sensitized by familial associations of rare disorders, realized that is was time to move beyond gene hunting and step into the individualized and aetiology-based cure (Josephson, Wiebe 2021; Nabbout, Kuchenbuch 2020; Thakran et al 2020). Thus, the gap between gene hunting and etiology-based personalized treatment is diminishing by the hour and the examples of applied precision medicine is growing. Identification of a precise genetic etiology can already direct physicians to prescribe treatments correcting specific metabolic defects avoid ASM drugs that can aggravate the pathogenic defect or select ASM that counteract the functional disturbance caused by the gene mutation.

In the rush for development of therapeutics for rare diseases, the Food and Drug Administration (FDA) issued some guidance to industry (references 17 to 19). Such guidelines emphasise the importance of having a welldelineated natural history, identifying relevant clinical outcomes, and identifying or developing appropriate outcome assessments. Translation of genetic causes into new or more targeted treatments depends on effective model systems that illuminate the underlying biology and contribute to the development of new drugscreening protocols.

The present review, focuses mainly on paediatric-onset epilepsies, where the identification of multiple aetiologies and the potential for early intervention provides the ideal environment for the implementation of a preventive precision medicine approach. We will discuss the therapeutic implications following a specific genetic diagnosis and the correlation between genetic findings, pathophysiological mechanism and tailored seizure treatment.

For academic purposes, current evidences have been divided into four subtypes according to the impact of such knowledge on real patient's treatment:

1. Clinical-based evidence of known ASM affecting seizure frequency either worsening or improving seizure frequency

2. Precision medicine: novel treatment strategies developed from pathophysiological knowledge

3. Precision medicine: aetiology-based preventive treatments and in progress treatments

Clinical-based evidence of known ASM modifying - either worsening or improving - seizure frequency

One of the first and best example of how the identification of the underling genetic aetiology guides treatment is that of Dravet Syndrome (DS) the most renowned and studied developmental and epileptic encephalopathy (DEE) (Mei et al 2019). Well before the genetic cause was identified, some clinical reports indicated that sodium channel blockers including lamotrigine (LTG) and carbamazepine (CBZ) should be avoided in patients with DS since they have the potential to determine an increase in seizure frequency evolving to status epilepticus in some patients (Guerrini et al, 1998; de Lange et al 2018). Following the discovery that DS is associated to mutations of the alpha1 subunit of the sodium channel (SCN1A) possibly causing loss of channel function, the clinical evidence that the administration of sodium channel blockers made seizure 
worse was partly explained (Claes et al 2001; Mantegazza, Marini 2010; Mei et al 2019). Although complete seizure control is rarely attainable, clinical studies have shown that the combination of valproic acid (VPA), clobazam (CLB) and stiripentol (STP) is the most effective in preventing seizures especially avoiding the evolution into status epilepticus, frequently observed in infants with DS (Ceulemans et al 2004; Wirrel et al 2018, Wirrel and Nabbout 2019). STP was associated with a greater than $50 \%$ reduction in convulsive seizure frequency in $71 \%$ of cases, when added to VPA and CLB and markedly reduced status epilepticus (Chiron et al 2000). STP has multiple mechanisms of action including increasing peak concentration and duration of action of CLB and a direct anti-convulsing activity likely due to enhancement of inhibitory, GABAergic neurotransmission (Fisher 2011).These agents are generally well tolerated, with few patients discontinuing for adverse effects.

A second straightforward example of seizures aggravation by sodium channel blockers is observed in the DEE related to HCN1 gene Gly39Asp mutation (Marini et al 2018). Two patients with complete genotypephenotype correlation also shared the response to ASM especially worsening of seizures frequency when treated with phenytoin (PHT) and lacosamide (LCS). Consistent with this clinical report from HCN1 patients the administration of LTG in the genetic knock-in mouse model resulted in a paradoxical induction of seizures and spiking induction in mutant animals (Bleakley et al 2021).

On the contrary, in some well-defined DEE including those associated toSCN2A and SCN8A gene mutations, seizures are fully controlled by CBZ and PHT (Numis et al 2014; Pisano et al 2015; Ohba et al 2014; Larsen et al 2015; Wolff et al 2017; Dilena et al 2017; Gardella, Moeller 2019). SCN2A mutations response to sodium channel blockers is related to missense mutations leading to a gain of channel function and seizure onset before age 3 months, severe phenotypes yet seizures are stopped with PHT and CBZ (Wolff et al 2017). The story of $S C N 2 A$ adds another level of complexity to take into account when treating a patient with a genetic epilepsy. In some patients, the choice of the best ASM medication is guided not only by knowledge of the mutated gene but also by its functional consequences.

Carbamazepine and PHT are also recommended as first-line drugs for the treatment of seizures in patients with KCNQ2-encephalopathy, including those presenting with status epilepticus (Numis et al 2014; Pisano et al 2015). Most patients achieve seizure freedom on these ASM and it has been suggested that early seizure control may also improve neurodevelopmental outcome (Pisano et al 2015).

The proline-rich transmembrane protein 2 (PRRT2) is a presynaptic transmembrane protein interacting with members of the SNARE complex, which enables synaptic vesicle fusion. Disease-causing variants in PRRT2 gene result in haploinsufficiency and are clinically expressed with infantile onset focal seizures that have a very benign outcome and disappear by age 2 years in otherwise normal infants. During childhood or adolescence, some patients may develop paroxysmal kinesigenic choreoathetosis (Chen et al 2011; Marini et al 2012). Additional rare manifestations include hemiplegic migraine and absence seizures (Marini et al 2012). Small doses of CBZ are very effective to suppress seizures and the movement disorder episodes (De Gusmao, Silveira-Moriyama 2019).

Tuberous sclerosis complex (TSC) is a multisystem disease caused by inactivating mutations in either TSC1 or TSC2 genes that are important components of the mTOR (mammalian target of rapamycin) pathway which regulates a variety of neuronal functions, including cell proliferation, survival, growth, and plasticity. TSC is a major cause of severe and drug-resistant epilepsy, with focal seizures and infantile spasms occurring in about $80 \%$ of TSC infants (Curatolo et al 2018). Vigabatrin (GVG), a conventional GABAergic ASM is the first line drug for infantile spasms of patients with TSC, is very effective and spasms disappear completely in most patients (see below in the section of preventive treatment).

Precision medicine in the epilepsies also has an equally important role in facilitating avoidance of adverse reactions as in maximising efficacy, as illustrated by a number of recent example including POLG1 mutations who might develop fatal hepatic failure when treated with valproate (Hynynen et al 2014). An additional example is that of the HLA-B*15:02 allele which is highly predictive of carbamazepine-induced StevensJohnson syndrome, a severe hypersensitivity reaction, in patients of Asian origin (Chung et al 2004). These 
examples clearly show how much clinicians and families have gained from the knowledge of the underling gene mutations in the therapeutically management of some epilepsies and syndromes. Most epilepsies for which we obtained greater pathophysiological insights are related to ion channel gene mutations and available drugs targeting ion channels are our initial treating choice.

Table 1, here below, summarizes the above reported examples

\begin{tabular}{|c|c|c|c|c|c|}
\hline & Gene & $\begin{array}{l}\text { Functional } \\
\text { effect }\end{array}$ & ASM & ASM effect & Reference \\
\hline \multirow{10}{*}{$\begin{array}{l}\text { Known ASM } \\
\text { modifying - } \\
\text { worsening or } \\
\text { improving- } \\
\text { seizure frequency }\end{array}$} & $K C N Q^{2}$ & $\begin{array}{l}\text { Dominant } \\
\text { negative effect }\end{array}$ & $\begin{array}{l}\text { Na channel } \\
\text { blockers (CBZ, } \\
\text { PHT) }\end{array}$ & Improvement & $\begin{array}{l}\text { Numis et al } \\
\text { 2014; Pisano et } \\
\text { al } 2015\end{array}$ \\
\hline & $S C N 2 A$ & GOF & $\begin{array}{l}\text { Na channel } \\
\text { blockers (CBZ, } \\
\text { PHT) }\end{array}$ & Improvement & $\begin{array}{l}\text { Wolff et al } \\
2017\end{array}$ \\
\hline & $S C N 8 A$ & Unknown & $\begin{array}{l}\text { Na channel } \\
\text { blockers (CBZ, } \\
\text { PHT) }\end{array}$ & Improvement & $\begin{array}{l}\text { Larsen et al } \\
2018\end{array}$ \\
\hline & $S C N 1 A$ & $\mathrm{LOF}$ & $\begin{array}{l}\text { Na sodium } \\
\text { channel blockers } \\
\text { (CBZ, PHT, } \\
\text { LTG) }\end{array}$ & Worsening & $\begin{array}{l}\text { Guerrini et al } \\
\text { 1998; de Lange } \\
\text { et al } 2018\end{array}$ \\
\hline & $S C N 1 A$ & LOF & VPA, CLB, STP & $\begin{array}{l}\text { 1st line } \\
\text { treatment }\end{array}$ & $\begin{array}{l}\text { Wirrel et al } \\
\text { 2017; Wirrel, } \\
\text { Nabbout } 2018\end{array}$ \\
\hline & PRRT2 & haploinsufficiency & $\mathrm{CBZ}$ & $\begin{array}{l}1^{\text {st }} \text { line } \\
\text { treatment }\end{array}$ & $\begin{array}{l}\text { De Gusmao, } \\
\text { Silveira- } \\
\text { Moriyama } \\
2019\end{array}$ \\
\hline & $H C N 1$ & $\mathrm{LOF}$ & $\begin{array}{l}\text { Na sodium } \\
\text { channel } \\
\text { blockers (CBZ, } \\
\text { PHT, LTG, } \\
\text { LCM) }\end{array}$ & Worsening & $\begin{array}{l}\text { Marini et al } \\
2018\end{array}$ \\
\hline & TSC1 \& TSC2 & Inactivating & GVG & Improving IS & $\begin{array}{l}\text { Curatolo et al } \\
2018\end{array}$ \\
\hline & $P O L G$ & - & VPA & $\begin{array}{l}\text { Acute liver } \\
\text { failure }\end{array}$ & $\begin{array}{l}\text { Hynynen et al } \\
2014\end{array}$ \\
\hline & $\begin{array}{l}H L A-B^{*} 15: 02 \\
\text { allele }\end{array}$ & & $\mathrm{CBZ}$ & $\begin{array}{l}\text { Stevens- } \\
\text { Johnson } \\
\text { syndrome in } \\
\text { Asian origin }\end{array}$ & $\begin{array}{l}\text { Chung et al } \\
2004\end{array}$ \\
\hline
\end{tabular}

$\mathrm{ASM}=$ antiseizure medication; $\mathrm{GOF}=$ gain of function; $\mathrm{LOF}=$ loss of function; $\mathrm{STP}=$ stiripentol; $\mathrm{CLB}=$ clobazam; Mut= mutation, IS = infantile spasms

\section{Precision medicine: novel treatment strategies developed from pathophysiological knowl-} edge

Advances in the identification of the underlying causes of epilepsies led to a novel therapeutic approach 
which targets the underlying pathophysiology causing seizures and other comorbidities (EpiPM consortium 2015). Several conditions can now benefit from a more tailored treatment using novel compounds or drugs aiming to counteract or resolve the pathological mechanism and restore the disrupted brain function (Mei et al 2017; Perucca P, Perucca E 2020; Nabbout, Kuchenbuch 2020). We can distinguish three different categories of therapy which are used in these individual treatment strategies:

\section{Substitutive therapies}

2. Therapies that modify cell-signalling pathways

3. Function-based therapies

\section{Substitutive therapies}

Substitutive therapies are currently used to treat disorders that are related to inherited metabolic diseases including epilepsies caused by glucose transporter type 1 deficiency (GLUT1) or by vitamin deficiencies. The GLUT1 deficiency syndrome is caused by haploinsufficiency of the SLC2A gene (solute carrier family 2, facilitated glucose transporter member 1) (De Vivo et al 1991; Klepper et al 2020). Low level of cerebral glucose, due to the impaired transport, are associated to a spectrum of symptoms from paroxysmal, often exercise induced, movement disorders to epilepsy often combined and with variable degree of intellectual disability (De Vivo et al 1991; Weber et al 2008; Suls et al 2009; Mullen et al 2010). For this hereditary neurometabolic disorder, early diagnosis and treatment with ketogenic diets (KD) is an important and individualizing treatment. The $\mathrm{KD}$, a high-fat, low protein, and low-carbohydrate diet provides ketone bodies and also have a mechanism of action through GABA synthesis, resulting in a neuroprotective effect and protecting from epileptogenesis (Klepper et al 2020). Keton bodies transported through the blood-brain barrier and used as an alternative energetic substrate.

Although inborn errors of metabolism do not represent the most common cause of seizures, their early identification is of utmost importance, since for some the substitutive therapeutic measures beyond common anti-epileptic drugs, is essential either to control seizures, or to decrease the risk of neurodegeneration (van Karnebeek et al 2018)

Amongst the vitamin responsive epilepsies pyridoxine and pyridoxal phosphate (PLP) deficiencies caused by homozygous or compound heterozygous mutations of the ALDH7A1 (antiquitin) and PNPO genes are relatively frequent (Plecko B 2013, Wilson et al 2019). Administration of either pyridoxine and/or PLP determine seizures resolution and improvement of the overall general condition with a lifelong dependency on vitamin B6 supply (Coughlin et al 2021).

Cerebral folate deficiency is characterized by low levels of 5-methyltetrahydrofolate (the active metabolite of folate) in the nervous system but normal folate metabolism in the rest of the body. It may be associated mutations in the FOLR1 gene encoding the folate receptor $\alpha$. The intracerebral deficiency leads to severe developmental delay, movement disorder, white matter changes, bilateral basal ganglia calcification, and drug-refractory epilepsy. Treatment includes initiation of folinic acid to correct 5- methyltetrahydrofolate CSF levels (Pope et al 2019).

Biotinidase enzyme deficiency is a rare, autosomal recessive neurometabolic disorder, classical clinical presentation includes recurrent seizures in the first few months of life. Testing for biotinidase enzyme activity in the peripheral blood is readily available and is part of neonatal screening at birth in developed countries. Genetic testing is confirmatory. Treatment is life-long biotin supplementation at 5-20 mg/d regardless of weight or age for both these disorders (Wolf 2012)

Early diagnosis is also important for the treatment of epilepsy caused by neurodegenerative diseases such as neuronal ceroid lipofuscinosis type 2 (CLN2). In 2017, enzyme replacement therapy with cerliponase alfa (recombinant human TPP1) has been approved for the treatment of CLN2 disease, a rare neurodegenerative paediatric disorder caused by autosomal recessive mutations in the TPP1 gene resulting in a deficiency of the lysosomal enzyme tripeptidyl peptidase 1 (TPP1) (Markham 2017). CLN2 is characterized by seizures between the age of 2 and 4 years, language delay, motor dysfunction and behavioural problems. Long-term 
outcome of patients with CLN2 has dramatically improved since the introduction of targeted therapy with recombinant human tripeptidyl peptidase (Schultz et al 2018, Specchio et al 2020). This treatment has been associated with a slowing or even stabilization of the deterioration in gait and language ability. Participants receiving this treatment have been followed-up for 3 years and this effect seems to be maintained over time (Schultz et al 2019).

\section{b. Therapies that modify signalling pathways are used to treat autoimmune epilepsy and epilepsies related to the mTOR pathway}

Rapamycin (sirolimus), a macrolide compound isolated in 1975 from Streptomyces hygroscopicus, and Everolimus, derived from rapamycin with more favourable pharmacokinetic characteristics, inhibit the mTOR pathway and so control cellular proliferation and tumour growth. Both sirolimus and everolimus have demonstrated efficacy for the treatment of multiple aspects of TSC including renal angiomyolipoma and SEGAs, (Franz et al 2013; 2016; Krueger et al 2013). The efficacy of everolimus in the treatment of seizures for TSC patients with refractory epilepsy has been demonstrated in some clinical trials (Kreuger et al 2018; French et al 2016).

\section{c. Function-based therapies}

Mutations falling upon genes involved in ion channel functions including sodium $(\mathrm{Na}+)$, calcium $(\mathrm{Ca}+)$, and potassium $\mathrm{K}+$ ) collectively are responsible for a considerable proportion of infantile and childhood onset drug resistant genetic epilepsies and DEE (El Achkar et al 2015; Parrini et al 2017; Stödberg et al 2020; Alsubaie et al 2020; Willimsky et al 2021). The functional mechanisms underlying Na+ channel ion genes including SCN1A, the most relevant epilepsy gene, SCN8A and SCN2A, and their implications for treatment selection has been highlighted in the previous section especially in relation to sodium-channel blockers ASM.

Potassium channels play an important role in regulation of neuronal excitability. Pathogenic variants have been identified in several potassium channel genes including KCNA2, KCNB1, KCNC1, KCND2, KCND3, KCNH1, KCNH2, KCNH5, KCNJ10, KCNMA1, KCNQ2, KCNQ3, and KCNT1 and have been associated with a variety of epilepsy phenotypes from mild to severe conditions (Weckhuysen et al 2012; Marini et al 2017; Corbett et al 2016; McTague A. et al 2018; Symonds JD, McTague 2020; Nikitin, Vinogradova 2021)

In the first section we discussed how, seizures related to KCNQ2mutations, might be suppressed by ASM acting as sodium channel blockers. Here we review novel therapeutic strategies based upon better understanding KCNQ2-DEE pathophysiological mechanisms. For instance retigabine is a selective Kv7.2/3 (voltage-dependent neuronal potassium channel) activator and antagonizes the functional impairment caused byKNCQ2 mutations leading to a channel loss of function (Orhan et al.2014, Miceli et al 2018). A multicentre study including 11 patients proved the clinical efficacy and usefulness of retigabine in the treatment of KCNQ2 encephalopathy (Millichap et al., 2016). Unfortunately, the retigabine preparation originally approved for the treatment of focal seizures has been withdrawn from the market. Recently, a case report showed that gabapentin might also be regarded as a precision therapy for epileptic encephalopathy due to KCNQ2 loss-of-function mutations (Soldovieri et al 2020). It should be emphasized that there are KCNQ2DEE caused by gain-of-function variants (Devaux et al., 2016; Millichap et al., 2017). Therefore, evaluation of the specific variant is important for treatment selection, because Kv7.2/3 activators such as retigabine and gabapentin could be aggravating when used in patients with gain-of- function mutations. Extensive research is ongoing to identify novel molecules endowed with different actions on potassium channels.

Heterozygous, mostly de novo, variants in KCNT1 gene, encoding the potassium channel KNa1.1, play a causative role in a wide spectrum of seizure disorders including epilepsy of infancy with migrating focal seizures (EIMFS), severe ADSHE (Barcia et al 2012; Heron et al 2012). All gene variants evaluated to date seem to lead to a gain-of-function phenotype, irrespective of the type of associated epilepsy (Barcia et al 2019; McTague et al 2018). Seizures associated with KCNT1-related epilepsies are often severe and drugresistant. Quinidine is a class Ia antiarrhythmic and antimalarial drug which operates as a partial antagonist of KCNT1 sodium-activated potassium channel. In 2014 Bearden et al reported the first clinical evidence that quinidine may be an effective medication in mutations causing a gain of function leading to constitutive 
activation with a pharmacologic inhibition of the channel (Bearden et al 2014). A patients with EIMFS carrying an activating mutation in KCNT1 during treatment with quinidine had a dramatic reduction in seizure frequency. Additional two patients carrying KCNT1 gain of function mutations were treated with quinidine that was able to reverse channel function leading to a seizure reduction (Milligan et al 2014). Furthermore, Mikati et al in 2015, described a reduction of $80 \%$ of seizure frequency in a 3-years -old male with EIMFS due to a de novo, gain of function, KCNT1 mutation (Mikati et al 2015). In the same paper they described a second patient, 11-years-old female with a de novo, gain of function KCNT1 mutation and nocturnal seizures, who did not respond to quinidine. Further reports of patients carrying KCNT1 mutations treated with quinidine without success are on record (Chong et al 2016; Barcia et al 2019; Mullen et al 2018; Borlot et al 2020). Thus, since the efficacy of quinidine is unclear and controversial its use in daily practice is at present not recommended and needs further evaluation.

Beside voltage-gated ion channel genes and mutations disrupting ion influx and efflux through cell membrane ultimately leading to an abnormal neuronal excitability, an unbalance between excitation and inhibition can also be caused by mutations of genes involved in neurotransmitter functions and homeostasis including their release, re-uptake and production.

For instance, glutamate-mediated excitatory neurotransmission is partly mediated by activation of NMDA receptors. These cation channel-receptors are made up of several subunits. Mutations of GRIN1, GRIN2A, GRIN2B, and GRIN2D genes, which encode the NMDA receptors subunits, have associated with epileptic phenotypes (Carvil et al 2013; Lemke et al 2013; Lesca et al 2013; Lemke et al 2014). Memantine is a non-competitive N-methyl-d-aspartate (NMDA) receptor antagonist currently used for the treatment of Alzheimer's disease, the drug seems to also have an anticonvulsant effect of in some epilepsy animal models (Ghasemi M, Schachter 2011) mechanism of action seems to be related to NMDA receptor activity. In 2014 Pierson and colleagues, reported a 6, 5 years old patient with epileptic encephalopathy due to a de novo variant in GRIN2A gene (Pierson et al 2014). Mutant NMDA receptors were expressed in Xenopus laevis oocytes and treated with memantine. Exposure to memantine was shown to restore receptor proper function. Following this successful experimental testing the boy was treated with memantine which to led to a dramatic reduction of seizure and an improvement in the interictal EEG recordings (Pierson et al 2014). Further patients carrying GRIN2D and GRIN2B mutations were also treated with memantine without the same exciting results ( $\mathrm{Li}$ et al 2016; Platzer et al 2017). Similarly to quinidine, the efficacy of memantine is unclear and its use in patients is at present not indicated.

Genes related to inhibitory GABAA neurotransmitters also play a key role in genetic epilepsies including genetic generalized epilepsies (GGE) and several DEE. Mutations in genes coding for subunits of GAABA including GABRG2; GABRA1; GABRB3, GABRD are included amongst the long list of epilepsy genes (Dibbens et al 2004; El Achkar et al 2015; Parrini et al 2018; May et al 2018; Stödberg et al 2020; Alsubaie et al 2020; Willimsky et al 2021). Vinpocetine is an alkaloid derived from the periwinkle plant its pharmacological effects of include vasodilation antioxidation, anti-inflammation, synaptic modulation, and antithrombosis properties (Kiss, Karpati 1996). A single patient with Lennox-Gastaut syndrome carrying a GABRB3 (c.905A>G; Y302C) missense mutation was supplemented with Vinpocetine leading to reduction of epileptiform activity on EEG recordings and improvement of cognitive, behavioral and language functions (Billakota et al 2019). Vinpocetine passes through the blood-brain barrier and inhibits voltage-gated calcium channels, glutamate receptors, and voltage-dependent Na-channels (Bönöczk et al 2000). Antiepileptiform effects of vinpocetine could also derive from the above, plus its inhibition of sodium channels and potentiation of GABA activity (Billakota et al 2019).

Lafora disease is a severe, autosomal recessive, progressive myoclonus epilepsy, caused by loss of function mutations in EPM2A or NHLRC1 genes, encoding laforin and malin (Minassian et al 1998; Chan et al 2003). Lafora disease is invariably fatal, typically within 10 years. The absences or either proteins results in abnormal glycogen metabolism, abnormally long branches are generated by glycogen synthase, leading to glycogen insolubility that precipitate, aggregates and accumulates to form the Lafora Bodies (Nitschke et al 2018). Metformin, the most commonly prescribed drug for type 2 diabetes mellitus, is an activator of 
AMP-induced kinase (AMPK). In mice and rats, metformin was shown to have positive effects on neuronal survival and seizure termination (Dulovic et al 2014; Ashabi et al 2014; Yang et al 2017). Studies in a mouse model of Lafora disease showed that metformin ameliorated neuropathological symptoms, reduced seizure susceptibility and slightly reduced the numbers of Lafora bodies (Berthier et al 2016; Sanchez- Elexpuru et al 2017). In 2019 Bisulli et al described the efficacy of metformin in Lafora Disease in reduction of seizure frequency and global clinical improvement (of twelve treated patients, three had a clinical response, which was temporary in two) underlining the neuroprotective action of metformin (Bisulli et al 2019).

'Girls clustering epilepsy', originally described as 'female only epilepsy with cognitive impairment', is a rare disorder combining late infantile onset of clusters of focal seizures, usually occurring during febrile episodes, with cognitive impairment and frequent autistic traits (Depienne et al 2009, Marini et al 2012; Kolc et al 2020). In 2008, Dibbens et al linked this disorder to PCDH19 gene mutations (Dibbens et al 2008). Functional studies showed that PCDH19 gene mutations lead to deficiency of the neurosteroid allopregnanolone, one of the most potent GABA-receptor modulators (Marini et al 2012; Tan et al 2015). These findings provide evidence for a role of neurosteroids in PCDH19-related epilepsy and create realistic opportunities for targeted therapeutic interventions. Ganaxolone, a synthetic methyl analogue of allopregnanolone, has already been used to treat epilepsy and status epilepticus related to various aetiologies in open-label pilot studies, with encouraging results in some patients (Pieribone et al 2007; Kerrigan et al 2000) however, no randomized studies are available (Lattanzi et al 2021).

Bi-allelic loss-of-function variants in GAD1 cause a syndromic infantile onset developmental and epileptic encephalopathy presenting around the second months of life with epileptic spasms or myoclonic seizures (Chatron et al 2020). The GAD1 gene encodes an enzyme that catalyses the conversion of glutamic acid to GABA. Mutations cause a loss the enzyme of function thus the impaired GABAergic inhibitory function and excess of excitation might underlie neuronal hyperexcitability and seizures. A combination of KD and GVG both enhancing GABA function and availability have been shown to improve clinical outcome in these patients (Chatron et al 2020, von Hardenberg et al 2020 and Neuray et al 2020).

Table 2, here below, summarizes the above reported evidences

Type of treatment

Novel treatment strategies developed from pathophysiological knowledge $a$. Substitutive therapies

b. Therapies that modify cell-signalli

c. Function-based therapies

\section{Precision medicine: aetiology-based preventive treatments and in progress treatments}

Moving towards the future that, for some conditions is already the present, we step into

- preventive therapy

- antisense oligonucleotides treatment

- gene therapy 
Let's explore and revise where we are at with preventive therapies and other innovative therapies acting of mRNAs and DNAs of mutated genes thus have a true potential of curing the disorder and preventing the occurrence of symptoms.

The best example for which there is a very good scientific basis is represented by GVG a GABAergic drug, recommended as the first line therapy for infantile spasms and/or focal seizures in the first year of life (Chiron et al 1997; Elterman et al 2001). Patients with TSC are at very high risk for developing infantile seizures including spasms and focal seizures and about two thirds of them evolve into refractory epilepsy (Curatolo et al 2018). Patients with TSC also are at high risk for neurodevelopmental disorders whose severity is strongly related to age at seizure onset and epilepsy severity (de Vries et al 2015; O'Callaghan et al 2004). Early targeted interventions increase the probability of seizure-freedom and may protect neurodevelopment (Jozwiak et al 2011). Indeed, the multicenter study named EPISTOP Trial, demonstrated that treatment with GVG as soon as EEG recordings show the occurrence of epileptiform activity delays the onset and the severity of epilepsy (Kotulska et al 2021). Thus, by reducing the risk of having infantile refractory seizures, physicians have the power of protecting motor and cognitive development.

Prenatal treatment with maternal pyridoxine supplementation is another example of preventive therapy in epilepsies since it has been demonstrated to improve neurological outcome in newborns with pyridoxine dependent epilepsy and antiquitin deficiency (Stockler et al 2010).

Antisense oligonucleotides (ASOs) are synthetic oligonucleotide or oligonucleotide analogs that are designed to bind to the RNA in order to modulate the function of the targeted RNA. ASOs represent a novel therapeutic strategy to treat neurodegenerative diseases and seizures of severe epileptic encephalopathies. The best example of this new therapeutic strategy is represented, outside epilepsy, by the antisense drug Nusinersen that has been approved for the treatment of spinal muscular atrophy (SMA). Additional specific ASOs drugs are also currently in development for the treatment of amyotrophic lateral sclerosis, Huntington's disease, and Alzheimer's disease (Bennet et al 2019).

In epilepsies, it has been suggested that ASOs targeting the $S C N 1 A$ channelopathy might improve not only seizure control, but also impact the co-morbidities associated with DS (Wirrel and Nabbout, in 2019). In a mouse model of DS, intra-cerebro-ventricular ASOs was demonstrate to reduce the incidence of electrographic seizures and sudden unexpected death in epilepsy (SUDEP) by increasing the expression of productive Scn1a transcript in the mouse brain (Han et al 2020). ASO treatment is in progress also in humans, indeed the recruitment process for patients in two age groups (aged 13 to 18 years and 2 to 12 years of age) has begun [https://dravetsyndromenews.com/; the MONARCH trial (NCT04442295) Stoke Therapeutics]. The purpose of this clinical trial is to assess the safety of single ascending dose of ASO/STK-001 in children and adolescents with DS. Treatment with STK-001 is expected to increase the level of productive SCN1A m RNA resulting in the elevated expression of the sodium channel Nav1.1 protein and restore physiological levels, which is reduced in patients with DS.

In mouse models of SCN8A encephalopathy with gain of function mutations an ASO injection reduced SCN8A transcript and determined delayed seizure onset and lethality (Lenk et al 2020). At the same time, a Scn1a+/- haploinsufficient mouse model of DS was also successfully treated, intra-cerebro-ventricular injections extended survival of Dravet syndrome mice from 3 weeks to $>5$ months (Lenk et al 2020).

A cure is urgently needed for Lafora disease a teenage-onset devastating progressive myoclonus epilepsy. Current treatment is very disappointing including metformin (see previous section). Antisense oligonucleotide (Gys1-ASO) targeting the mRNA in the brain was administered by intra-cerebro-ventricular injection in the mouse model. Treatment determined a reduction of Lafora body formation (Ahonen et al 2021). The Gys1ASO could be administered to patients via lumbar puncture thus reach the brain and be effective in also in patients.

Ataluren is an available drug, at present prescribed in patients with Duchenne muscular dystrophy (Morkous 2020), which suppresses premature stop codons caused by nonsense gene mutations thus enabling full-length protein. A current clinical trial reached its Phase 2 (randomized, double-blind, placebo- 
controlled clinical trial, NCT02758626) in testing the safety and efficacy on seizure types and frequency of the drug in DS with nonsense mutation or cyclin-dependent kinase-like 5 (CDKL5) deficiency, (https://clinicaltrials.gov/ct2/show/NCT02758626).

For more than 10 years, gene therapy for neurological diseases has experienced intensive research development. Most trials are still in pre-clinical phase 1 and 2. Delivery is the major issue for central nervous system therapies in general, and particularly for gene therapy, the blood brain barrier restricts the passage of vectors thus strategies to bypass this obstacle are a central focus of research. Gene therapy products can be tailored to solve the pathophysiological mechanisms, including the use of gene replacement, gene silencing, transplicing, modulation of cellular pathways to improve phenotype or expression of suicide gene (Piguet et al 2021). There are only few gene therapies approved for the management of neurological disorders including two for SMA (Zolgensma- Novartis and Spinraza- Biogen) (Servais et al 2021).

For epilepsy the gene therapy approach is still very much a challenge. NPY is a neuropeptide expressed in the brain, where it acts as a neuromodulator, affecting pathways that range from cellular to circuit level. In the context of epilepsy, NPY is thought to act as an endogenous anticonvulsant, indeed its overexpression in the brain with the aid of viral vectors can suppress seizures in animal models of epilepsy. Therefore, NPY-based gene therapy may represent a novel approach for the treatment of patients with epilepsies (Cattaneo et al 2021). Wickham et al in 2019 demonstrate that NPY application, in hippocampal slices surgically resected from patients with drug-resistant TLE, significantly reduces chemically induced epileptiform activity in the dentate gyrus (Wickham et al 2019). Increasing the levels of NPY could be an alternative approach to achieve a therapeutic effect and suppress seizure activity.

Gene therapy has delivered promising results in animal experimental models raising the hopes that it might soon become available for patients. CRISPR/Cas9 biotechnology holds great promise in neurological therapy, pending the clearance of major delivery, efficiency, and specificity hurdles (Gumusgoz et al 2021).

Table 3, here below, summarizes the above reported evidences

\begin{tabular}{lll}
\hline & type of treatment & gene \\
\hline Aetiology-based preventive treatments and in progress treatments & Preventive teraphy & TSC1/TS \\
& & Pyridoxin \\
SCN1A \\
nonsense \\
Clinical ongoing trial & CDKL5 d \\
& & SCN8A \\
& In progress (tested only in animal models) & SCN1A \\
& & EPM2A/H \\
\hline
\end{tabular}

$\mathrm{ASO}=$ antisense oligonucleotide; $\mathrm{sz}=$ seizure

\section{Conclusions}

The evidences and examples discussed in previous sections clearly indicates that identifying the pathogenic variant in individual patients with genetic epilepsies is relevant not only for diagnosis and prognosis, but also for patient's management including selection of the best medication and/or the indication of which drugs might worsen seizure frequency or cause severe side effects thus should be avoided. Research is now very sensitive to the urgent need to identify a cure for the disorder/diseases improving seizures and accompanying comorbidities.

Furthermore, our increased understanding of the aetiologies of epilepsies, in some patients, allowed to identify specific targets for therapies that go beyond ASM and that enable treatment of the cause of epilepsy. We are already witnessing a major shift in our paradigm of epilepsy treatment and have moved towards the era of therapies that target the underlying cause and mechanisms of epilepsy. Available, commercialised 
and approved by the authorities, aetiology-based treatments are only partially satisfying, several drugs have proven effective in some patients and not in others carrying similar genotypes. Results are discordant and not univocal for most of the newly proposed, precision medicines. Moreover such treatments are still focused on stopping seizures.

There is no doubt that gene therapy will change our therapeutic approach to monogenic epilepsies. To reverse the pathophysiological impact of pathogenic variants, gene editing seems to be a very promising tool and is likely to be most effective when administered during the early stages of disease or even preventively. In this scenario, the challenge for epileptologists is to identify the causes of epilepsy early in order to promote preventive therapies and to avoid the occurrence of epilepsy, including seizures and comorbidities.

In conclusion the whole scientific community is rapidly evolving towards a more curative, pathophysiologybased and preventive therapeutic approach of disorders featuring seizures and comorbidities. Meanwhile, there are three main requirements for a systematic approach to precision medicine in epilepsy: first of all is patient's registry, a key requirement to enrol large cohorts of individuals with epilepsy who have been phenotypically and genomically characterized; second a standardized functional characterization of mutations in each of the epilepsy genes and third, since we are dealing with rare disorders, multi-center, randomized, controlled trials are needed and feasible when functional studies identifies new targets that might be translated into personalized and tailored drugs. Reaching these goals depends on the development of collaborative and integrated research groups that bring together researchers with clinical, genetic, and biological expertise. Physicians and families are ready and looking forward to this new era of precision medicine.

References

1. Scheffer IE, Berkovic S, Capovilla G, et al. ILAE classification of the epilepsies: Position paper of the ILAE Commission for Classification and Terminology. Epilepsia. 2017;58(4):512-521.

2. Ragona F, Granata T, Dalla Bernardina B, et al.Cognitive development in Dravet syndrome: a retrospective, multicenter study of 26 patients. Epilepsia. 2011;52(2):386-92

3. Caraballo RH, Cejas N, Chamorro N, Kaltenmeier MC, Fortini S, Soprano AM. Landau-Kleffner syndrome: a study of 29 patients. Seizure. 2014;23(2):98-104.

4. Meldrum BS, Horton RW. Physiology of status epilepticus in primates. Arch Neurol 1973;28:1-9.

5. Trinka E, Cock H, Hesdorffer D et al. A definition and classification of status epilepticus-Report of the ILAE Task Force on Classification of Status Epilepticus. Epilepsia. 2015;56(10):1515-23.

6. Mei D, Parrini E, Marini C, Guerrini R. The Impact of Next-Generation Sequencing on the Diagnosis and Treatment of Epilepsy in Paediatric Patients. Mol Diagn Ther. 2017;21(4):357-373.

7. Dunn P, Albury CL, Maksemous N, et al Next Generation Sequencing Methods for Diagnosis of Epilepsy Syndromes. Front Genet. 2018; 9: 20.

8. Nabbout R, Kuchenbuch M. Impact of predictive, preventive and precision medicine strategies in epilepsy. Nat Rev Neurol. 2020;16(12):674-688.

9. Parrini E, Marini C, Mei D et al. Diagnostic Targeted Resequencing in 349 Patients with DrugResistant Pediatric Epilepsies Identifies Causative Mutations in 30 Different Genes. Hum Mutat. 2017;38(2):216-225

10. Hebbar M, Mefford HC. Recent advances in epilepsy genomics and genetic testing. F1000Resaerch 2020, 12;9:F1000 Faculty Rev-185.

11. Stödberg T, Tomson T, Barbaro M, et al. Epilepsy syndromes, etiologies, and the use of next-generation sequencing in epilepsy presenting in the first 2 years of life: A population-based study. Epilepsia. 2020;61(11):2486-2499.

12. Symonds JD, McTague. Epilepsy and developmental disorders: Next generation sequencing in the clinic. A. Eur J Paediatr Neurol. 2020;24:15-23

13. Guerrini R, Noebels J. How can advances in epilepsy genetics lead to better treatments and cures? Adv Exp Med Biol. 2014;813:309-17.

14. Szepetowski P. Genetics of human epilepsies: Continuing progress. Presse Med. 2018;47(3):218-226 
15. Josephson CB, Wiebe S. Precision Medicine: Academic dreaming or clinical reality? Epilepsia. 2021;62 Suppl 2:S78-S89.

16. Thakran S, Guin D, Singh P, et al. Genetic Landscape of Common Epilepsies: Advancing towards Precision in Treatment. Int J Mol Sci. 2020;21(20):7784

17. FDA. Workshop on natural history studies of rare diseases. 2012. Bethesda, MD: FDA. Rare diseases: natural history studies for drug development guidance for industry. In: Administration FD, ed. 2019. https://www.fda.gov/media/122425/download

18. US Food and Drug Administration. Human gene therapy for rare diseases, guidance for industry (FDA, 2020).

19. FDA (Food and Drug Administration). Application of current statutory authorities to human somatic cell therapy products and gene therapy products. Fed. Regist. 58, 53248-53251 (1993).

20. Mei D, Cetica V, Marini C, Guerrini R. Dravet syndrome as part of the clinical and genetic spectrum of sodium channel epilepsies and encephalopathies. Epilepsia. 2019;60 Suppl 3:S2-S7.

21. Guerrini R, Dravet C, Genton P, Belmonte A, Kaminska A, Dulac O. Lamotrigine and seizure aggravation in severe myoclonic epilepsy. Epilepsia. 1998;39:508-12.

22. de Lange IM, Gunning B, Sonsma ACM et al. Influence of contraindicated medication use on cognitive outcome in Dravet syndrome and age at first afebrile seizure as a clinical predictor in SCN1A-related seizure phenotypes. Epilepsia. 2018;59(6):1154-1165

23. Claes L, Del-Favero J, Ceulemans B, Lagae L, Van Broeckhoven C, De Jonghe P. De novo mutations in the sodium-channel gene SCN1A cause severe myoclonic epilepsy of infancy. Am J Hum Genet. 2001;68(6):1327-32.

24. Marini C, Mantegazza M. Na+ channelopathies and epilepsy: recent advances and new perspectives. Expert Rev Clin Pharmacol. 2010;3(3):371-84.

25. Ceulemans B, Boel M, Claes L, Dom L, Willekens H, Thiry P, Lagae L. Severe myoclonic epilepsy in infancy: toward an optimal treatment. J Child Neurol. 2004 Jul;19(7):516-21

26. Wirrell EC, Laux L, Donner E, et al. Optimizing the Diagnosis and Management of Dravet Syndrome: Recommendations From a North American Consensus Panel. Pediatr Neurol. 2017;68:18-34.e3.

27. Wirrell EC, Nabbout R Recent Advances in the Drug Treatment of Dravet Syndrome. CNS Drugs. 2019;33(9):867-881.

28. Chiron C, Marchand MC, Tran A, et al. Stiripentol in severe myoclonic epilepsy in infancy: a randomised placebo-controlled syndrome-dedicated trial. STICLO study group. Lancet. 2000;356:1638-42.

29. Fisher JL. The effects of stiripentol on GABAA receptors. Epilepsia 2011;52:76-78

30. Marini C, Porro A, Rastetter A et al. HCN1 mutation spectrum: from neonatal epileptic encephalopathy to benign generalized epilepsy and beyond. Brain. 2018;141(11):3160-3178.

31. Bleakley LE, McKenzie CE, Soh et al. Cation leak underlies neuronal excitability in an HCN1 developmental and epileptic encephalopathy. Brain. 2021. Online ahead of print.

32. Numis AL, Angriman M, Sullivan JE, et al. KCNQ2 encephalopathy: delineation of theelectroclinical phenotype and treatment response. Neurology. 2014;82:368-70.

33. Pisano T, Numis AL, Heavin SB et al. Early and effective treatment of KCNQ2 encephalopathy. Epilepsia. 2015;56:685-91.

34. Ohba C, Kato M, Takahashi S et al. Early onset epileptic encephalopathy caused by de novo SCN8A mutations. Epilepsia 2014:55, 994-1000

35. Larsen J, Carvill GL, Gardella E et al. The phenotypic spectrum of SCN8A encephalopathy. Neurology. 2015;84(5):480-9.

36. Wolff M, Johannesen KM, Hedrich UB et al. Genetic and phenotypic heterogeneity suggest therapeutic implications in SCN2A-related disorders. Brain 2017;140, 1316-1336

37. Gardella E, Møller RS. Phenotypic and genetic spectrum of SCN8A-related disorders, treatment options, and outcomes. Epilepsia. 2019;60 Suppl 3:S77-S85.

38. Dilena, R. Striano P, Gennaro E, et al. Efficacy of sodium channel blockers in SCN2A early infantile epileptic encephalopathy. Brain Dev. 2017;39, 345-348

39. Chen WJ, Lin Y, Xiong ZQ, et al. Exome sequencing identifies truncating mutations in PRRT2 that 
cause paroxysmal kinesigenic dyskinesia. Nat Genet 2011;43:1252-1255.

40. Marini C, Conti V, Mei D, et al. PRRT2 mutations in familial infantile seizures, paroxysmal dyskinesia, and hemiplegic migraine. Neurology. 2012;79(21):2109-14.

41. De Gusmao CM, Silveira-Moriyama L. Paroxysmal movement disorders - practical update on diagnosis and management. Expert Rev Neurother. 2019;19(9):807-822.

42. Curatolo P, Nabbout R, Lagae L et al. Management of epilepsy associated with tuberous sclerosis complex: updated clinical recommendations. Eur J Paediatr Neurol. 2018;22(5):738-48.

43. Hynynen J, Komulainen T, Tukiainen E, et al. Acute liver failure after valproate exposure in patients with POLG1 mutations and the prognosis after liver transplantation. Liver Transpl. 2014; 20:1402-12.

44. Chung WH, Hung SI, Hong HS, et al. Medical genetics: a marker for Stevens-Johnson syndrome. Nature. 2004; 428:486.

45. EpiPM consortium. A road Map for precision medicine in the epilepsies. Lancet Neurol. 2015 December ; 14(12): 1219-122

46. Perucca P, Perucca E. Identifying mutations in epilepsy genes: Impact on treatment selection. Epilepsy Res. 2019 May;152:18-30

47. De Vivo DC, Trifiletti RR, Jacobson RI, et al. Defective glucose transport across the blood-brain barrier as a cause of persistent hypoglycorrhachia, seizures, and developmental delay. N Engl J Med. 1991;325(10):703-709.

48. Klepper J, Akman C, Armeno M et al. Glut1 Deficiency Syndrome (Glut1DS): State of the art in 2020 and recommendations of the international Glut1DS study group. Epilepsia Open. 2020;5(3):354-365.

49. Weber YG, Storch A, Wuttke TV, et al. GLUT1 mutations are a cause of paroxysmal exertion-induced dyskinesias and induce hemolytic anemia by a cation leak. J Clin Invest. Jun 2008;118(6):2157-2168.

50. Suls A, Mullen SA, Weber YG, et al. Early-onset absence epilepsy caused by mutations in the glucose transporter GLUT1. Ann Neurol. 2009;66(3):415-419.

51. Mullen SA, Suls A, De Jonghe P, Berkovic SF, Scheffer IE. Absence epilepsies with widely variable onset are a key feature of familial GLUT1 deficiency. Neurology. 2010;75(5):432-440.

52. van Karnebeek CDM, Sayson B, Lee JJY et al. Metabolic Evaluation of Epilepsy: A Diagnostic Algorithm With Focus on Treatable Conditions.Front Neurol. 2018;9:1016.

53. Plecko B. Pyridoxine and pyridoxlaphosphate-dependent epilepsies. Handb Clin Neurol. 2013;113:18117.

54. Wilson MP, Plecko B, Mills PB, Clayton PT. Disorders affecting vitamin B6 metabolism. J Inherit Metab Dis. 2019 Jul;42(4):629-646.

55. Coughlin CR, Tseng LA, Abdenur JE,et al. Consensus guidelines for the diagnosis and management of pyridoxine-dependent epilepsy due to alpha-aminoadipic emialdehyde dehydrogenase deficiency. J Inherit Metab Dis. 2021;44(1):178-192.

56. Pope S, Artuch R, Heales S, Rahman S. Cerebral folate deficiency: analytical tests and differential diagnosis. J Inherit Metab Dis. 2019;42:655-72.

57. Wolf B. Biotinidase deficiency: "If you have to have an inherited metabolic disease, this is the one to have." Genet Med. 2012;14: 565-75.

58. Markham A. Cerliponase Alfa: First Global Approval. Drugs. 2017;77(11):1247-1249.

59. Schulz, A, Ajayi T, Specchio N, et al. Study of intraventricular cerliponase alfa for CLN2 disease. N. Engl. J. Med. 2018;378:1898-1907

60. Specchio N, Pietrafusa N, Trivisano M. Changing Times for CLN2 Disease: The Era of Enzyme Replacement Therapy. Ther Clin Risk Manag. 2020;16:213-222.

61. Schulz, A. et al. Persistent treatment effect of cerliponase alfa in children with CLN2 disease: a 3 year update from an ongoing multicenter extension study. Mol. Genet. Metab. 2019;126:S133

62. Franz DN, Belousova E, Sparagana S et al. Efficacy and safety of everolimus for subependymal giant cell astrocytomas associated with tuberous sclerosis complex (EXIST-1): a multicentre, randomised, placebo-controlled phase 3 trial. Lancet 2013; 381:125-32.

63. Franz DN, Belousova E, Sparagana S et al. Long-term use of everolimus in patients with tuberous sclerosis complex: final results from the EXIST-1 Study. PLoS ONE;2016;11:e0158476. 
64. Krueger DA, Care MM, Agricola K, Tudor C, Mays M, Franz DN. Everolimus long-term safety and efficacy in subependymal giant cell astrocytoma. Neurology 2013;80:574-80.

65. French JA, Lawson JA, Yapici Z, et al. Adjunctive everolimus therapy for treatment-resistant focalonset seizures associated with tuberous sclerosis (EXIST-3): a phase 3, randomised, double-blind, placebo-controlled study. Lancet 2016; 388, 2153-2163

66. Krueger DA, Capal JK, Curatolo P, et al. Short-term safety of mTOR inhibitors in infants and very young children with tuberous sclerosis complex (TSC): Multicentre clinical experience. Eur J Paediatr Neurol. 2018;22(6):1066-1073.

67. El Achkar CM, Olson HE, Poduri A, Pearl PL. The genetics of the epilepsies. Curr Neurol Neurosci Rep. 2015;15(7):39.

68. Stödberg T, Tomson T, Barbaro M, et al. Epilepsy syndromes, etiologies, and the use of next-generation sequencing in epilepsy presenting in the first 2 years of life: A population-based study. Epilepsia. 2020;61(11):2486-2499

69. Alsubaie L, Aloraini T, Amoudi M, et al. Genomic testing and counselling: The contribution of nextgeneration sequencing to epilepsy genetics. Ann Hum Genet. 2020;84(6):431-436

70. Willimsky EK, Munzig A, Mayer K, et al. Next Generation Sequencing in Pediatric Epilepsy Using Customized Panels: Size Matters. Neuropediatrics. 2021;52(2):92-97

71. Weckhuysen S, Mandelstam S, Suls A et al. KCNQ2 encephalopathy: emerging phenotype of a neonatal epileptic encephalopathy. Ann Neurol. 2012;71(1):15-25.

72. Marini C, Romoli M, Parrini E, et al. Clinical features and outcome of 6 new patients carrying de novo KCNB1 gene mutations. Neurol Genet. 2017;3(6):e206.

73. Corbett MA, Bellows ST, Li M, et al. Dominant KCNA2 mutation causes episodic ataxia and pharmacoresponsive epilepsy. Neurology. 2016;87(19):1975-1984

74. McTague A, Nair U, Malhotra S, et al. Clinical and molecular characterization of KCNT1-related severe early-onset epilepsy. Neurology. 2018;90(1):e55-e66.

75. Symonds JD, McTague A. Epilepsy and developmental disorders: Next generation sequencing in the clinic. Eur J Paediatr Neurol. 2020;24:15-23

76. Nikitin ES, Vinogradova LV. Potassium channels as prominent targets and tools for the treatment of epilepsy. Expert Opin Ther Targets. 2021:1-13.

77. Orhan G, Bock M, Schepers D, et al. Dominant-negative effects of KCNQ2 mutations are associated with epileptic encephalopathy, Ann Neurol 2014;75:382-94.

78. Miceli F, Soldovieri MV, Ambrosino P, et al Pharmacological Targeting of Neuronal Kv7.2/3 Channels: A Focus on Chemotypes

and Receptor Sites, Curr Med Chem 2018; 25:2637-60.

1. Millichap JJ, Park KL, Tsuchida T et al. KCNQ2 encephalopathy: Features, mutational hot spots, and ezogabine treatment of 11 patients. Neurol Genet. 2016 22;2(5):e96

2. Soldovieri MV, Freri E, Ambrosino P et al. Gabapentin treatment in a patient with KCNQ2 developmental epileptic encephalopathy. Pharmacol Res. 2020;160:105200.

3. Devaux J, Abidi A, Roubertie A et al. A Kv7.2 mutation associated with early onset epileptic encephalopathy with suppression-burst enhances Kv7/M channel activity. Epilepsia. 2016;57(5):e87-93.

4. Millichap JJ, Miceli F, De Maria M, et al. Infantile spasms and encephalopathy without preceding neonatal seizures caused by KCNQ2 R198Q, a gain-of-function variant. Epilepsia. 2017;58(1):e10-e15.

5. Barcia G, Fleming MR, Deligniere A et al. De novo gain-of-function KCNT1 channel mutations cause malignant migrating partial seizures of infancy. Nat Genet. 2012;44(11):1255-9.

6. Heron SE, Smith KR, Bahlo M, et al. Missense mutations in the sodium-gated potassium channel gene KCNT1 cause severe autosomal dominant nocturnal frontal lobe epilepsy. Nat Genet 2012;44:11881190.

7. Barcia G, Chemaly N, Kuchenbuch M et al. Epilepsy with migrating focal seizures: KCNT1 mutation hotspots and phenotype variability. Neurol Genet. 2019;5(6):e363.

8. Bearden D, Strong A, Ehnot J, DiGiovine M, Dlugos D, Goldberg EM. Targeted treatment of migrating 
partial seizures of infancy with quinidine.Ann Neuro 2014;76: 457-461. 14.

9. Milligan CJ, Li M, Gazina EV, Heron SE et al. KCNT1 gain of function in 2 epilepsy phenotypes is reversed by quinidine. Ann Neurol. 2014;75(4):581-90.

10. Mikati MA, JiangYH, Carboni M, et al.Quinidine in the treatment of KCNT-positive epilepsies. Ann Neurol 2015;78:995-999.

11. Chong PF, Nakamura R, Saitsu H, Matsumoto N, Kira R. Ineffective quinidine therapy in early onset epileptic encephalopathy with KCNT1 mutation. Ann Neurol 2016;79:502-503.

12. Mullen SA, Carney PW, Roten A et al. Precision therapy for epilepsy due to KCNT1 mutations: A randomized trial of oral quinidine. Neurology. 2018;90(1):e67-e72.

13. Borlot F, Abushama A, Morrison-Levy N et al. KCNT1-related epilepsy: An international multicenter cohort of 27 pediatric cases. Epilepsia. 2020;61(4):679-692.

14. Carvill GL, Regan BM, Yendle SC, et al. GRIN2A mutations cause epilepsy-aphasia spectrum disorders. Nat Genet 2013;45:1073-6.

15. Lemke JR, Lal D, Reinthaler EM, et al. Mutations in GRIN2A cause idiopathic focal epilepsy with rolandic spikes. Nat Genet 2013;45:1067-72.

16. Lesca G, Rudolf G, Bruneau N, et al. GRIN2A mutations in acquired epileptic aphasia and related childhood focal epilepsies and encephalopathies with speech and language dysfunction. Nat Genet $2013 ; 45: 1061-6$.

17. Lemke JR, Hendrickx R, Geider K, et al. GRIN2B mutations in West syndrome and intellectual disability with focal epilepsy. Ann Neurol 2014;75:147-54.

18. Ghasemi M, Schachter SC. The NMDA receptor complex as a therapeutic target in epilepsy: a review. Epilepsy Behav. 2011;22(4):617-40.

19. Pierson TM, Yuan H, Marsh ED, et al. GRIN2A mutation and early-onset epileptic encephalopathy: personalized therapy with memantine. Ann Clin Transl Neurol 2014;1:190-8.

20. Li D, Yuan H, Ortiz-Gonzalez XR et al. GRIN2D recurrent de novo dominant mutation causes a severe epileptic encephalopathy treatable with NMDA receptor channel blockers. Am J Hum Genet 2016;99:802-16.

21. Platzer K, Yuan H, Schutz H, et al. GRIN2B encephalopathy: novel findings on phenotype, variant clustering, functional consequences and treatment aspects. J Med Genet. 2017;54(7):460-470

22. May P, Girard S, Harrer M et al. Rare coding variants in genes encoding GABAA receptors in genetic generalised epilepsies: an exome-based case-control study. Lancet Neurol. 2018;17(8):699-708.

23. Dibbens LM, Feng HJ., Richards $M$ et al. GABRD encoding a protein for extra- or peri-synaptic GABA-A receptors is a susceptibility locus for generalized epilepsies. Hum. Molec. Genet. 2004:13: 1315-1319

24. Kiss B, Karpati E. Mechanism of action of vinpocetine. Acta Pharm Hung. 1996;66(5):213-24.

25. Billakota S, Andresen JM, Gay BC, et al. Personalized medicine: Vinpocetine to reverse effects of GABRB3 mutation. Epilepsia. 2019;60:2459- 2465.

26. Bonoczk P, Gulyas B, Adam-Vizi V, et al. Role of sodium channel inhibition in neuroprotection: effect of vinpocetine. Brain Res Bull. 2000;53(3):245-54.

27. Minassian BA, Lee JR, Herbrick JA, et al. Mutations in a gene encoding a novel protein tyrosine phosphatase cause progressive myoclonus epilepsy. Nat. Genet. 1998, 20, 171-174

28. Chan EM, Young EJ, Ianzano L, Munteanu I et al. Mutations in NHLRC1 cause progressive myoclonus epilepsy. Nat. Genet. 2003:35,125-127

29. Nitschke F, Ahonen SJ, Nitschke S, Mitra S, Minassian BA. Lafora disease - from pathogenesis to treatment strategies. Nat Rev Neurol. 2018;14(10):606-617

30. Dulovic M, Jovanovic M, Xilouri M, et al. The protective role of AMP- activated protein kinase in alpha- synuclein neurotoxicity in vitro. Neurobiol. Dis 2014;63:1-11

31. Ashabi G, Khodagholi F, Khalaj L, Goudarzvand M. \& Nasiri, M. Activation of AMP- activated protein kinase by metformin protects against global cerebral ischemia in male rats: interference of AMPK/PGC-1 $\alpha$ pathway. Metab. Brain Dis. 2014;29:47-58

32. Yang Y. Zhu B, Zheng F, et al. Chronic metformin treatment facilitates seizure termination. Biochem. 
Biophys. Res. Commun. 2017;484: 450-455

33. Berthier A. Payá M, García-Cabrero AM et al. Pharmacological interventions to ameliorate neuropathological symptoms in a mouse model of Lafora disease. Mol. Neurobiol. 53, 1296-1309 (2016).

34. Sanchez- Elexpuru G, Serratosa JM, Sanz P. \& Sanchez MP. 4-PBA and metformin decrease sensitivity to PTZ- induced seizures in a malin knockout model of Lafora disease. Neuroreport 28, 268-271 (2017).

35. Bisulli F, Muccioli L, d'Orsi G, et al. Treatment with metformin in twelve patients with Lafora disease. Orphanet J Rare Dis. 2019 21;14(1):149.

36. Dibbens LM, Tarpey PS, Hynes K, et al. X-linked protocadherin 19 mutations cause female-limited epilepsy and cognitive impairment. Nat Genet. 2008;40:776-781

37. Depienne C, Bouteiller D, Keren B et al. Sporadic infantile epileptic encephalopathy caused by mutations in PCDH19 resembles dravet syndrome but mainly affects females. PLoS Genet. 2009 5(2):e1000381

38. Kolc KL, Sadleir LG, Depienne C et al. A standardized patient-centered characterization of the phenotypic spectrum of PCDH19 girls clustering epilepsy. Transl Psychiatry. 2020;10(1):127-

39. Marini C, Darra F, Specchio N. Focal seizures with affective symptoms are a major feature of PCDH19 gene-related epilepsy. Epilepsia. 2012;53(12):2111-9.

40. Tan C, Shard C, Ranieri E et al. Mutations of protocadherin 19 in female epilepsy (PCDH19-FE) lead to allopregnanolone deficiency. Hum Mol Genet 2015;24:5250-9

41. Pieribone VA, Tsai J, Soufflet C et al. Clinical evaluation of ganaxolone in pediatric and adolescent patients with refractory epilepsy. Epilepsia. 2007;48:1870-4. 98.

42. Kerrigan JF, Shields WD, Nelson TY et al. Ganaxolone for treating intractable infantile spasms: a multicenter, open-label, add-on trial. Epilepsy Res. 2000;42:133-9.

43. Lattanzi S, Riva A, Striano P. Ganaxolone treatment for epilepsy patients: from pharmacology to place in therapy. Expert Rev Neurother. 2021:1-16.

44. Chatron N, Becker F, Morsy H et al. Bi-allelic GAD1 variants cause a neonatal onset syndromic developmental and epileptic encephalopathy. Brain. 2020;143(5):1447-1461

45. von Hardenberg S, Richter MF et al. Rational therapy with vigabatrin and a ketogenic diet in a patient with GAD1 deficiency. Brain. 2020;143(11):e91.

46. Neuray C, Maroofian R, Scala M, et al. Early-infantile onset epilepsy and developmental delay caused by bi-allelic GAD1 variants. Brain 2020; 143: 2388-97.

47. Chiron C, Dumas C, Jambaque I, et al. Randomized trial comparing vigabatrin and hydrocortisone in infantile spasms due to tuberous sclerosis. Epilepsy Res. 1997;26:389-395.

48. Elterman RD, Shields WD, Mansfield KA, Nakagawa J. US Infantile Spasms Vigabatrin Study Group. Randomized trial of vigabatrin in patients with infantile spasms. Neurology. 2001;57:1416-1421.

49. de Vries PJ, Whittemore VH, Leclezio L, et al. Tuberous sclerosis associated neuropsychiatric disorders (TAND) and the TAND Checklist. Pediatr Neurol. 2015;52:25-35.

50. O'Callaghan FJK, Harris T, Joinson C, et al. The relation of infantile spasms, tubers, and intelligence in tuberous sclerosis complex. Arch Dis Child. 2004; 89:530-533.

51. Jozwiak S, Kotulska K, Domaniska-Pakiela D, et al. Antiepileptic treatment before the onset of seizures reduces epilepsy severity and risk of mental retardation in infants with tuberous sclerosis complex. Eur J Paediatr Neurol. 2011;15:424-431.

52. Kotulska K, Kwiatkowski DJ, Curatolo P, et al Prevention of Epilepsy in Infants with Tuberous Sclerosis Complex in the EPISTOP Trial. Ann Neurol. 2021;89(2):304-314.

53. Stockler S, Plecko B, Gospe SM Jr et al. Pyridoxine dependent epilepsy and antiquitin deficiency: clinical and molecular characteristics and recommendations for diagnosis, treatment and follow-up. Mol Genet Metab. 2011;104(1-2):48-60.

54. Bennett CF, Krainer AR, Cleveland DW. Antisense Oligonucleotide Therapies for Neurodegenerative Diseases. Annu Rev Neurosci. 2019;42:385-406.

55. Wirrell EC, Nabbout R. Recent Advances in the Drug Treatment of Dravet Syndrome. CNS Drugs. 2019;33(9):867-881.

56. Han Z, Chen C, Christiansen A, et al. Antisense oligonucleotides increase Scn1a expression and reduce seizures and SUDEP incidence in a mouse model of Dravet syndrome. Sci Transl Med. 
2020;12(558):eaaz6100.

57. Lenk GM, Jafar-Nejad P, Hill SF et al. Scn8a Antisense Oligonucleotide Is Protective in Mouse Models of SCN8A Encephalopathy and Dravet Syndrome. Ann Neurol. 2020 Mar;87(3):339-346.

58. Ahonen S, Nitschke S, Tamar R et al. Gys1 antisense therapy rescues neuropathological bases of murine Lafora disease. https://doi.org/10.1101/2021.02.11.430846

59. Morkous SS. Treatment with Ataluren for Duchene Muscular Dystrophy. Pediatr Neurol Briefs. 2020;34:12.

60. Gene Therapy Clinical Trials Worldwide. Hippocampal NPY gene transfer in subjects with Intractable Temporal Lobe Epilepsy. Abedia.com http://www.abedia.com/ wiley/record_detail.php?ID=1758 (2004).

61. Piguet F, de Saint Denis T, Audouard E et al. The Challenge of Gene Therapy for Neurological Diseases: Strategies and Tools to Achieve Efficient Delivery to the Central Nervous System. Hum Gene Ther. 2021; online ahead of print.

62. Servais L, Baranello G, Scoto M, Daron A, Oskoui M. Therapeutic interventions for spinal muscular atrophy: preclinical and early clinical development opportunities. Expert Opin Investig Drugs. 2021 Apr 13:1-9.

63. Cattaneo S, Verlengia G, Marino P Simonato M, Bettegazzi B. NPY and Gene Therapy for Epilepsy: How, When,... and Y. Front Mol Neurosci 2021;13:608001. doi: 10.3389/fnmol.2020.608001.

64. Wickham, J. et al. Inhibition of epileptiform activity by neuropeptide Y in brain tissue from drugresistant temporal lobe epilepsy patients. Sci. Rep. 9, 19393 (2019). 137.

65. Gumusgoz E, Guisso DR, Kasiri S, et al. Targeting Gys1 with AAV-SaCas9 Decreases Pathogenic Polyglucosan Bodies and Neuroinflammation in Adult Polyglucosan Body and Lafora Disease Mouse Models Neurotherapeutics. 2021 Apr 8. Online ahead of print. 